\title{
HOMFLY polynomials of torus links as generalized Fibonacci polynomials
}

\author{
Kemal Taşköprü \\ Department of Mathematics \\ Faculty of Arts and Sciences \\ Bilecik Şeyh Edebali University \\ Bilecik, 11000, Turkey \\ kemal.taskopru@bilecik.edu.tr
}

\author{
İsmet Altıntaş \\ Department of Mathematics \\ Faculty of Arts and Sciences \\ Sakarya University \\ Sakarya, 54187, Turkey \\ ialtintas@sakarya.edu.tr
}

Submitted: Jun 11, 2015; Accepted: Sep 29, 2015; Published: Oct 16, 2015

Mathematics Subject Classifications: 57M25, 11B39, 11C08

\begin{abstract}
The focus of this paper is to study the HOMFLY polynomial of $(2, n)$-torus link as a generalized Fibonacci polynomial. For this purpose, we first introduce a form of generalized Fibonacci and Lucas polynomials and provide their some fundamental properties. We define the HOMFLY polynomial of $(2, n)$-torus link with a way similar to our generalized Fibonacci polynomials and provide its fundamental properties. We also show that the HOMFLY polynomial of $(2, n)$-torus link can be obtained from its Alexander-Conway polynomial or the classical Fibonacci polynomial. We finally give the matrix representations and prove important identities, which are similar to the Fibonacci identities, for the our generalized Fibonacci polynomial and the HOMFLY polynomial of $(2, n)$-torus link.
\end{abstract}

Keywords: HOMFLY polynomial; Alexander-Conway polynomial; torus link; Fibonacci polynomial; Binet's formula; Fibonacci identities

\section{Introduction}

An important part of works of the knot theory is about discovering the knot invariants and determining the type of knot by means of the knot invariants.

A knot polynomial is a knot invariant whose coefficients encode some of the properties of a given knot. There are important knot polynomials in literature [1,2,5,7,11,14-18]. The first of these was defined by J. W. Alexander in 1928 [1]. Let $\mathcal{L}$ denote an oriented link (or knot) diagram. The Alexander polynomial, $\Delta_{\mathcal{L}}(t)$, is a Laurent polynomial in the variable $t$ with integer coefficients associated with the link diagram $\mathcal{L}$ in an invariant way. There are several ways to define and calculate the Alexander polynomial, see $[1,8,10,21]$. 
In 1969, J. Conway defined a version of the Alexander polynomial with a skein relation, usually referred to as the Alexander-Conway polynomial [7]. The Alexander-Conway polynomial, $\nabla_{\mathcal{L}}(z)$, is a Laurent polynomial in the variable $z$ with integer coefficients associated with the oriented link diagram $\mathcal{L}$. $\nabla_{\mathcal{L}}(z)$ is an ambient isotopy invariant of the link $\mathcal{L}$ determined by the following axioms:

$$
\begin{aligned}
\nabla_{\mathcal{L}_{+}}(z)-\nabla_{\mathcal{L}_{-}}(z) & =z \nabla_{\mathcal{L}_{0}}(z) \\
\nabla_{\bigcirc}(z) & =1
\end{aligned}
$$

where $\mathcal{L}_{+}, \mathcal{L}_{-}$and $\mathcal{L}_{0}$ are skein diagrams drawn in Figure 1 and $\bigcirc$ is any diagram of the unknot.

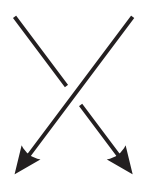

$\mathcal{L}_{+}$

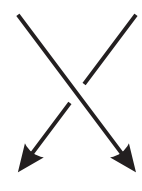

$\mathcal{L}_{-}$

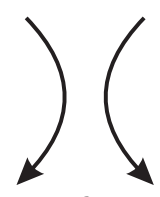

$\mathcal{L}_{0}$

Figure 1: Skein Diagrams

The relationship between the Alexander-Conway polynomial and the original Alexander polynomial is given by

$$
\Delta_{\mathcal{L}}(t)=\nabla_{\mathcal{L}}\left(t^{1 / 2}-t^{-1 / 2}\right)
$$

Here, $\Delta_{\mathcal{L}}(t)$ must be properly normalized (by multiplication $\pm t^{n / 2}$ ) to satisfy the skein relation

$$
\begin{aligned}
\Delta_{\mathcal{L}_{+}}(t)-\Delta_{\mathcal{L}_{-}}(t) & =\left(t^{1 / 2}-t^{-1 / 2}\right) \Delta_{\mathcal{L}_{0}}(t) \\
\Delta_{\bigcirc}(t) & =1 .
\end{aligned}
$$

Note that this relation gives a Laurent polynomial in the variable $t^{1 / 2}$.

The significance of the skein relation was not realized until the early 1980s. In 1984, V. Jones discovered a new polynomial invariant for knots and links [14]. The Jones polynomial, $V_{\mathcal{L}}(t)$, is a Laurent polynomial in the variable $t^{1 / 2}$ associated with the oriented link diagram $\mathcal{L}$. $V_{\mathcal{L}}(t)$ is an ambient isotopy invariant of the link $\mathcal{L}$ determined by the following axioms:

$$
\begin{aligned}
t^{-1} V_{\mathcal{L}_{+}}(t)-t V_{\mathcal{L}_{-}}(t) & =\left(t^{1 / 2}-t^{-1 / 2}\right) V_{\mathcal{L}_{0}}(t) \\
V_{\bigcirc}(t) & =1 .
\end{aligned}
$$

The Jones polynomial leds to discover more knot polynomials, such as so-called HOMFLY polynomial $[5,11]$. The HOMFLY polynomial is a generalization of the AlexanderConway polynomial and the Jones polynomial. The HOMFLY polynomial, $P_{\mathcal{L}}(a, z)$, is 
two variables Laurent polynomial for the oriented link diagram $\mathcal{L} . P_{\mathcal{L}}(a, z)$ is an ambient isotopy invariant of the link $\mathcal{L}$ determined by the following axioms:

$$
\begin{aligned}
a^{-1} P_{\mathcal{L}_{+}}(a, z)-a P_{\mathcal{L}_{-}}(a, z) & =z P_{\mathcal{L}_{0}}(a, z) \\
P_{\bigcirc}(a, z) & =1 .
\end{aligned}
$$

The HOMFLY polynomial specializes to the Jones polynomial for $a=t$ and $z=$ $t^{1 / 2}-t^{-1 / 2}$, the Alexander-Conway polynomial for $a=1$ and the Alexander polynomial for $a=1$ and $z=t^{1 / 2}-t^{-1 / 2}$.

From the axioms (1) and (2), we have $a^{-1}-a=z P_{\bigcirc} \circ$ or $\delta=\left(a^{-1}-a\right) z^{-1}$ with $\delta=P_{\bigcirc}$, where $\bigcirc \bigcirc$ is trivial link with two components. So, we get $\delta=-t^{1 / 2}-t^{-1 / 2}$ for the Jones polynomial and $\delta=0$ for the Alexander-Conway polynomial.

To easily calculate the HOMFLY polynomial, we can write the equality (1) as follows:

$$
\begin{aligned}
& P_{\mathcal{L}_{+}}=a z P_{\mathcal{L}_{0}}+a^{2} P_{\mathcal{L}_{-}} \\
& P_{\mathcal{L}_{-}}=-a^{-1} z P_{\mathcal{L}_{0}}+a^{-2} P_{\mathcal{L}_{+}} .
\end{aligned}
$$

Some of the properties of the HOMFLY polynomial are listed as follows.

1. If $\bigcirc_{\mu}$ is a trivial $\mu$-component link, then $P_{\bigcirc_{\mu}}(a, z)=\delta^{\mu-1}$.

2. $P_{-\mathcal{L}}(a, z)=P_{\mathcal{L}}(a, z)$, where $-\mathcal{L}$ is reverse oriented $\mathcal{L}$.

3. $P_{\mathcal{L}^{*}}(a, z)=P_{\mathcal{L}}\left(a^{-1},-z\right)$, where $\mathcal{L}^{*}$ is the mirror image of $\mathcal{L}$.

4. $P_{\mathcal{L}_{1} \sharp \mathcal{L}_{2}}(a, z)=P_{\mathcal{L}_{1}}(a, z) P_{\mathcal{L}_{2}}(a, z)$, where $\mathcal{L}_{1} \sharp \mathcal{L}_{2}$ is composition of $\mathcal{L}_{1}$ and $\mathcal{L}_{2}$

5. $P_{\mathcal{L}_{1} \sqcup \mathcal{L}_{2}}(a, z)=\delta P_{\mathcal{L}_{1}}(a, z) P_{\mathcal{L}_{2}}(a, z)$, where $\mathcal{L}_{1} \sqcup \mathcal{L}_{2}$ is disjoint union of $\mathcal{L}_{1}$ and $\mathcal{L}_{2}$

It is well known that the Alexander-Conway polynomial of $(2, n)$-torus link is the classical Fibonacci polynomial, see $[15,19]$. The theory and application of the Fibonacci numbers and Fibonacci polynomials attract attention in the modern science. There are many studies on the Fibonacci polynomial and its generalization, see $[3,6,20,22,23]$ and others.

This paper is organized as follows. Section 2 includes summary information about our version of generalized Fibonacci and Lucas polynomial and its fundamental properties.

In section 3, we show that the HOMFLY polynomial of $(2, n)$-torus link is a generalized Fibonacci polynomial and examine its fundamental properties. We also give the relationship between this polynomial and the classical Fibonacci polynomial. Thus, we can obtain the HOMFLY polynomial of $(2, n)$-torus link from its Alexander-Conway polynomial and the Jones polynomial of the same link from its Alexander polynomial.

In the last section, we work on the matrix representations of polynomials examined. We prove identities similar to the identities of Catalan, Cassini and d'Ocagne which are important Fibonacci identities. 


\section{A generalization of Fibonacci polynomials}

In this section, we introduce the following form of generalized Fibonacci and Lucas polynomials which will be the basis to study the HOMFLY polynomials of $(2, n)$-torus links and their properties.

Definition 1. The generalized Fibonacci polynomials $\left\{F_{n}(a, z)\right\}_{n=0}^{\infty}$ in two variables $a, z$ are defined by the recurrence relation

$$
F_{n}(a, z)=a z F_{n-1}(a, z)+a^{2} F_{n-2}(a, z), \quad n \geqslant 2
$$

with initial conditions

$$
F_{0}(a, z)=0, \quad F_{1}(a, z)=1 .
$$

For $a=1$, we obtain Fibonacci polynomials in $z$ defined by the recurrence relation

$$
f_{n}(z)=z f_{n-1}(z)+f_{n-2}(z), \quad n \geqslant 2
$$

with initial conditions

$$
f_{0}(z)=0, \quad f_{1}(z)=1
$$

It is not difficult to see that

$$
F_{n}(a, z)=a^{n-1} f_{n}(z) .
$$

In the polynomial sequence $\left\{F_{n}(a, z)\right\}$, we have Fibonacci numbers for $a=z=1$ and natural numbers for $a=i$ and $z=-2 i$ with $i^{2}=-1$.

If $a=i q^{1 / 2}$ and $z=-i\left(q^{1 / 2}+q^{-1 / 2}\right)$, then the q-form of $F_{n}(a, z)$ is

$$
F_{n}(q)=1+q+q^{2}+\ldots+q^{n-1} .
$$

The generating function of the sequence $\left\{F_{n}(a, z)\right\}$ is defined by

$$
g_{F}(\lambda)=\sum_{n=0}^{\infty} F_{n}(a, z) \lambda^{n} .
$$

The characteristic equation of the relation (5) is

$$
\lambda^{2}-a z \lambda-a^{2}=0
$$

and the roots are

$$
\alpha=\frac{a z+a \sqrt{z^{2}+4}}{2}, \quad \beta=\frac{a z-a \sqrt{z^{2}+4}}{2} .
$$

We have the following relations between $a, z$ and $\alpha, \beta$ :

$$
\alpha+\beta=a z, \quad \alpha \beta=-a^{2}, \quad \alpha-\beta=a \sqrt{z^{2}+4} .
$$


Definition 2. The generalized Lucas polynomials in two variables $a, z$ are defined by the recurrence relation

$$
L_{n}(a, z)=a z L_{n-1}(a, z)+a^{2} L_{n-2}(a, z), \quad n \geqslant 2
$$

with initial conditions

$$
L_{0}(a, z)=2, \quad L_{1}(a, z)=a z .
$$

For $a=1$, we have Lucas polynomials defined by the recurrence relation

$$
l_{n}(z)=z l_{n-1}(z)+l_{n-2}(z), \quad n \geqslant 2
$$

with initial conditions

$$
l_{0}(z)=2, \quad l_{1}(z)=z .
$$

It is easily seen that

$$
L_{n}(a, z)=a^{n-1} l_{n}(z) .
$$

The characteristic equation of $L_{n}(a, z)$ and its roots are the same as $F_{n}(a, z)$ 's.

We now give some fundamental properties of the polynomials $F_{n}(a, z)$ and $L_{n}(a, z)$. We provide the properties of $F_{n}(a, z)$ and leave the proof of the properties of $L_{n}(a, z)$ for readers.

Proposition 3. The generating functions of $F_{n}(a, z)$ and $L_{n}(a, z)$ are

$$
g_{F}(\lambda)=\frac{\lambda}{1-a z \lambda-a^{2} \lambda^{2}}
$$

and

$$
g_{L}(\lambda)=\frac{2-a z \lambda}{1-a z \lambda-a^{2} \lambda^{2}} .
$$

Proof. The generating function of $F_{n}(a, z)$ has the following form:

$$
g_{F}(\lambda)=F_{0}(a, z)+F_{1}(a, z) \lambda+F_{2}(a, z) \lambda^{2}+\ldots
$$

After the multiplications $a z \lambda g_{F}(\lambda)$ and $a^{2} \lambda^{2} g_{F}(\lambda)$, we have

$$
\begin{aligned}
\left(1-a z \lambda-a^{2} \lambda^{2}\right) g_{F}(\lambda)= & F_{0}(a, z)+\left(F_{1}(a, z)-a z F_{0}(a, z)\right) \lambda \\
& +\sum_{n=2}^{\infty}\left(F_{n}(a, z)-a z F_{n-1}(a, z)-a^{2} F_{n-2}(a, z)\right) \lambda^{n} \\
= & \lambda .
\end{aligned}
$$

Thus, we obtain the generating function of $F_{n}(a, z)$.

By using rational expansion theorem [13], the Binet's formulas for the polynomials $F_{n}(a, z)$ and $L_{n}(a, z)$ are obtained from Proposition 3 as follows. 
Proposition 4. For $n \geqslant 0$, the Binet's formulas of $F_{n}(a, z)$ and $L_{n}(a, z)$ are

$$
F_{n}(a, z)=\frac{\alpha^{n}-\beta^{n}}{\alpha-\beta}
$$

and

$$
L_{n}(a, z)=\alpha^{n}+\beta^{n} .
$$

Proposition 5. For $n \geqslant 1$, the explicit formulas of $F_{n}(a, z)$ and $L_{n}(a, z)$ are given by

$$
F_{n}(a, z)=\sum_{k=0}^{\left\lfloor\frac{n-1}{2}\right\rfloor}\left(\begin{array}{c}
n-k-1 \\
k
\end{array}\right) a^{n-1} z^{n-2 k-1}
$$

and

$$
L_{n}(a, z)=\sum_{k=0}^{\left\lfloor\frac{n}{2}\right\rfloor} \frac{n}{n-k}\left(\begin{array}{c}
n-k \\
k
\end{array}\right) a^{n} z^{n-2 k} .
$$

Proof. By arranging the identity 1.60 in [12] for $n$, we get

$$
\sum_{k=0}^{\left\lfloor\frac{n-1}{2}\right\rfloor}\left(\begin{array}{c}
n-k-1 \\
k
\end{array}\right)(x y)^{k}(x+y)^{n-2 k-1}=\frac{x^{n}-y^{n}}{x-y} .
$$

In this identity, by writing $\alpha$ and $\beta$ instead of $x$ and $y$, respectively and using the equalities (8) and (10), we obtain explicit formula of $F_{n}(a, z)$.

Proposition 6. For $n \geqslant 1$, there are the following identities between $F_{n}(a, z)$ and $L_{n}(a, z)$ :

$$
\begin{aligned}
& F_{n}(a, z)=\frac{L_{n+1}(a, z)+a^{2} L_{n-1}(a, z)}{a^{2}\left(z^{2}+4\right)} \\
& L_{n}(a, z)=F_{n+1}(a, z)+a^{2} F_{n-1}(a, z) .
\end{aligned}
$$

Proof. Proof follows from the Binet's formulas (10) and (11).

\section{HOMFLY polynomials of $(2, n)$-torus links}

Let $\mathcal{L}_{n}$ be an oriented diagram for $(2, n)$-torus link, where diagram has the right-hand orientation, see Figure 2. For simplicity, let $P_{n}(a, z)$ denote the HOMFLY polynomial $P_{\mathcal{L}_{n}}(a, z)$.

Theorem 7. The HOMFLY polynomial of $(2, n)$-torus link provides the following recurrence relation:

$$
P_{n}(a, z)=a z P_{n-1}(a, z)+a^{2} P_{n-2}(a, z), \quad n \geqslant 2 .
$$


Proof. Let us apply the skein operation to a designated crossing of oriented diagram $\mathcal{L}_{n}$ drawn in Figure 2 of $(2, n)$-torus link. If we change over-crossing to under-crossing or vice versa over the designated crossing of the $\mathcal{L}_{n}$, the resulting diagram is a $(2, n-2)$-torus link by II. Reidemeister moves. If we separate the same crossing, then the resulting diagram is a $(2, n-1)$-torus link. From the equality (3), we have

$$
P_{n}(a, z)=a z P_{n-1}(a, z)+a^{2} P_{n-2}(a, z)
$$

for $n \geqslant 2$. So the proof is complete.

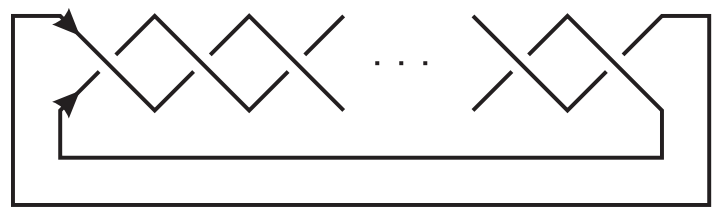

Figure 2: $(2, n)$-Torus Link

The recurrence relation in Theorem 7 is also provided for the Jones polynomial with $a=t$ and $z=t^{1 / 2}-t^{-1 / 2}$, Alexander-Conway polynomial with $a=1$ and Alexander polynomial with $a=1$ and $z=t^{1 / 2}-t^{-1 / 2}$.

Now, let's rewrite the Theorem 7 as a recurrence relation with initial conditions.

Definition 8. The HOMFLY polynomials $\left\{P_{n}(a, z)\right\}_{n=0}^{\infty}$ for the oriented diagrams of $(2, n)$-torus links is defined by the recurrence relation

$$
P_{n}(a, z)=a z P_{n-1}(a, z)+a^{2} P_{n-2}(a, z), \quad n \geqslant 2
$$

with initial conditions

$$
P_{0}(a, z)=\delta=\left(a^{-1}-a\right) z^{-1}, \quad P_{1}(a, z)=1 .
$$

Then, $P_{n}(a, z)$ is a generalized bivariate Fibonacci polynomial. For $a=1$ in (13) and (14) we obtain the classical Fibonacci polynomial, $f_{n}(z)$, or the Alexander-Conway polynomial, $\nabla_{n}(z)$, for $(2, n)$-torus link.

From the third property of the HOMFLY polynomials in section 1 and the identity (4), the HOMFLY polyomial sequence $\left\{P_{n}(a, z)\right\}$ can be applied to $\mathcal{L}_{n}^{*}$ which is mirror image of $(2, n)$-torus link $\mathcal{L}_{n}$. Hence, by using $P_{-n}(a, z)$ instead of $P_{\mathcal{L}_{n}^{*}}(a, z)$, we have

$$
P_{-n}(a, z)=-a^{-1} z P_{-(n-1)}(a, z)+a^{-2} P_{-(n-2)}(a, z) .
$$

The characteristic equation of the recurrence relation (13) is

$$
\lambda^{2}-a z \lambda-a^{2}=0
$$

and the roots are

$$
\alpha=\frac{a z+a \sqrt{z^{2}+4}}{2}, \quad \beta=\frac{a z-a \sqrt{z^{2}+4}}{2} .
$$


Note that, the characteristic equations of the recurrence relations for the polynomials $P_{n}(a, z) F_{n}(a, z)$ and $L_{n}(a, z)$ are same and also the roots of the characteristic equation of the recurrence relation (15) are $\frac{1}{\alpha}$ and $\frac{1}{\beta}$.

By using (13) and (14), we give the following HOMFLY polynomials table of $(2, n)$ torus links.

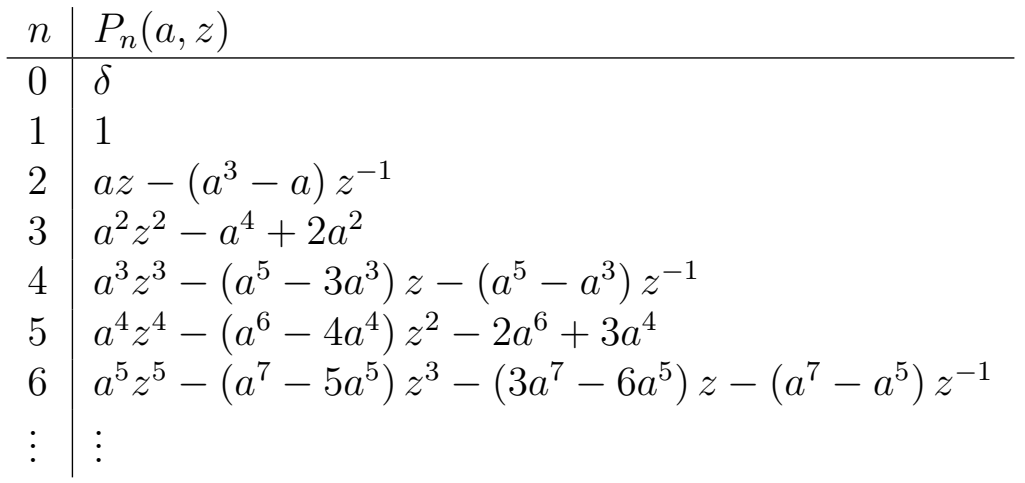

We now prove some properties of the polynomial $P_{n}(a, z)$.

Proposition 9. The generating function

$$
g_{P}(\lambda)=\sum_{n=0}^{\infty} P_{n}(a, z) \lambda^{n}
$$

of the sequence $\left\{P_{n}(a, z)\right\}$ is given by

$$
g_{P}(\lambda)=\frac{a^{3} z \lambda-a^{2}+1}{-a^{3} z \lambda^{2}-a^{2} z^{2} \lambda+a z} .
$$

Proof. The generating function of $P_{n}(a, z)$ has the following form:

$$
g_{P}(\lambda)=P_{0}(a, z)+P_{1}(a, z) \lambda+P_{2}(a, z) \lambda^{2}+\ldots
$$

After the multiplications $a z \lambda g(\lambda)$ and $a^{2} \lambda^{2} g(\lambda)$, we have

$$
\begin{aligned}
\left(1-a z \lambda-a^{2} \lambda^{2}\right) g_{P}(\lambda)= & P_{0}(a, z)+\left(P_{1}(a, z)-a z P_{0}(a, z)\right) \lambda \\
& +\sum_{n=2}^{\infty}\left(P_{n}(a, z)-a z P_{n-1}(a, z)-a^{2} P_{n-2}(a, z)\right) \lambda^{n} \\
= & P_{0}(a, z)+\left(P_{1}(a, z)-a z P_{0}(a, z)\right) \lambda .
\end{aligned}
$$

We thus obtain the generating function of $P_{n}(a, z)$ as

$$
g(\lambda)=\frac{P_{0}(a, z)+\left(P_{1}(a, z)-a z P_{0}(a, z)\right) \lambda}{1-a z \lambda-a^{2} \lambda^{2}}
$$

or

$$
g(\lambda)=\frac{a^{3} z \lambda-a^{2}+1}{-a^{3} z \lambda^{2}-a^{2} z^{2} \lambda+a z} .
$$


Proposition 10. The Binet's formula for the HOMFLY polynomial sequence $\left\{P_{n}(a, z)\right\}$ is given by

$$
P_{n}(a, z)=A \alpha^{n}+B \beta^{n}
$$

where

$$
A=\frac{\left(1+a^{2}\right) z+\left(1-a^{2}\right) \sqrt{z^{2}+4}}{2 a z \sqrt{z^{2}+4}}
$$

and

$$
B=-\frac{\left(1+a^{2}\right) z-\left(1-a^{2}\right) \sqrt{z^{2}+4}}{2 a z \sqrt{z^{2}+4}} .
$$

Proof. By using rational expansion theorem, [13], we get Binet's form from (17) as

$$
P_{n}(a, z)=(1-\beta \delta) \frac{\alpha^{n}}{\alpha-\beta}-(1-\alpha \delta) \frac{\beta^{n}}{\alpha-\beta} .
$$

By using (16) and (8) in (18) and reformulating, we reach Binet's formula.

Proposition 11. For $n \geqslant 2$, the explicit formula for the HOMFLY polynomial of $(2, n)$ torus link is given by

$$
\begin{aligned}
P_{n}(a, z)= & \sum_{k=0}^{\left\lfloor\frac{n-1}{2}\right\rfloor}\left(\begin{array}{c}
n-k-1 \\
k
\end{array}\right) a^{n-1} z^{n-2 k-1} \\
& +\left(1-a^{2}\right) \sum_{k=0}^{\left\lfloor\frac{n-2}{2}\right\rfloor}\left(\begin{array}{c}
n-k-2 \\
k
\end{array}\right) a^{n-1} z^{n-2 k-3} .
\end{aligned}
$$

Proof. Likewise the proof of Proposition 5, by using identity 1.60 in [12] and considering the equations (8) and (18), we reach the explicit formula.

Theorem 12. The relation between the HOMFLY polynomial $P_{n}(a, z)$ of $(2, n)$-torus link and generalized Fibonacci polynomial $F_{n}(a, z)$ is

$$
P_{n}(a, z)=F_{n}(a, z)+\left(a-a^{3}\right) z^{-1} F_{n-1}(a, z), \quad n \geqslant 2 .
$$

Proof. Proof follows from the equalities (12) and (19).

The following theorem lets us to obtain the HOMFLY polynomial of $(2, n)$-torus link from its Alexander-Conway polynomial.

Theorem 13. Let $P_{n}(a, z)$ and $\nabla_{n}(z)$ denote the HOMFLY polynomial and the Alexander -Conway polynomial for $(2, n)$-torus link, respectively. Then, for $n \geqslant 2$,

$$
P_{n}(a, z)=a^{n-1}\left(\nabla_{n}(z)+\left(1-a^{2}\right) z^{-1} \nabla_{n-1}(z)\right) .
$$


Proof. By using (6) in the relation (20),

$$
P_{n}(a, z)=a^{n-1}\left(f_{n}(z)+\left(1-a^{2}\right) z^{-1} f_{n-1}(z)\right) .
$$

Since $f_{n}(z)=\nabla_{n}(z)$, the proof is completed.

Corollary 14. Let $V_{n}(t)$ and $\Delta_{n}(t)$ denote the Jones polynomial and the Alexander polynomial for $(2, n)$-torus link, respectively. Then, for $n \geqslant 2$,

$$
V_{n}(t)=t^{n-1}\left(\Delta_{n}(t)-\left(t^{3 / 2}+t^{1 / 2}\right) \Delta_{n-1}(t)\right)
$$

Proof. If we take $a=t$ and $z=t^{1 / 2}-t^{-1 / 2}$ in the identity (21), we obtain the equality (22).

\section{Matrix representations}

In this section, we use the sum property for determinants of the matrices which are associated with the generalized Fibonacci polynomial and the HOMFLY polynomial of $(2, n)$-torus link to prove the following identities. For similar studies, see $[4,6,9,24]$.

Theorem 15. Let

$$
\boldsymbol{A}=\left[\begin{array}{ll}
a z & 1 \\
a^{2} & 0
\end{array}\right]
$$

be a matrix which is associated with $F_{n}(a, z)$. For $n \geqslant 1$,

$$
\boldsymbol{A}^{n}=\left[\begin{array}{cc}
F_{n+1} & F_{n} \\
a^{2} F_{n} & a^{2} F_{n-1}
\end{array}\right]
$$

with $F_{n}=F_{n}(a, z)$.

Proof. We proceed by induction on $n$. The theorem holds for $n=1$. We assume that it holds for $n=k(k \geqslant 1)$. Then, we have

$$
\begin{aligned}
\mathbf{A}^{k+1} & =\left[\begin{array}{cc}
F_{k+1} & F_{k} \\
a^{2} F_{k} & a^{2} F_{k-1}
\end{array}\right]\left[\begin{array}{cc}
a z & 1 \\
a^{2} & 0
\end{array}\right] \\
& =\left[\begin{array}{cc}
F_{k+2} & F_{k+1} \\
a^{2} F_{k+1} & a^{2} F_{k}
\end{array}\right] .
\end{aligned}
$$

Corollary 16 (Cassini's Identity). For $n \geqslant 1$,

$$
F_{n+1} F_{n-1}-F_{n}^{2}=(-1)^{n} a^{2 n-2} .
$$


Proof. From the determinant of $n$-th power of the matrix A, we have $\left|\mathbf{A}^{n}\right|=(-1)^{n} a^{2 n}$. On the other hand, the determinant of matrix (23) is $\left|\mathbf{A}^{n}\right|=a^{2}\left(F_{n+1} F_{n-1}-F_{n}^{2}\right)$. Hence, we obtain the identity (24).

Corollary 17. For $n, m \geqslant 0$,

$$
F_{n+m+1}=F_{n+1} F_{m+1}+a^{2} F_{n} F_{m}
$$

and in particular, for $n=m$,

$$
F_{2 n+1}=F_{n+1}^{2}+a^{2} F_{n}^{2}
$$

Proof. These results are obtained from $\mathbf{A}^{n+m}=\mathbf{A}^{n} \mathbf{A}^{m}$.

Theorem 18 (d'Ocagne's Identity). For $m \geqslant n \geqslant 1$,

$$
F_{n+1} F_{m}-F_{n} F_{m+1}=(-1)^{n} a^{2 n} F_{m-n}
$$

Proof. Let $\mathbf{B}_{0}$ be the matrix

$$
\mathbf{B}_{0}=\left[\begin{array}{cc}
F_{n+2} & F_{n+2} \\
a^{2} F_{n+1} & a^{2} F_{n+1}
\end{array}\right] .
$$

We construct the matrix $\mathbf{B}_{1}$ by adding the first column of the matrix $\mathbf{A}^{n}$ multiplied with $a^{2}$ to the first column of the matrix $\mathbf{B}_{0}$ multiplied with $a z$. Hence, we have

$$
\mathbf{B}_{1}=\left[\begin{array}{cc}
F_{n+3} & F_{n+2} \\
a^{2} F_{n+2} & a^{2} F_{n+1}
\end{array}\right] .
$$

In the same way, we construct the matrix $\mathbf{B}_{r}$ by adding the first column of the matrix $\mathbf{B}_{r-2}$ multiplied with $a^{2}$ to the first column of the matrix $\mathbf{B}_{r-1}$ multiplied with $a z$, for $r \geqslant 2$. Thus, we have

$$
\mathbf{B}_{2}=\left[\begin{array}{cc}
F_{n+4} & F_{n+2} \\
a^{2} F_{n+3} & a^{2} F_{n+1}
\end{array}\right], \quad \mathbf{B}_{3}=\left[\begin{array}{cc}
F_{n+5} & F_{n+2} \\
a^{2} F_{n+4} & a^{2} F_{n+1}
\end{array}\right],
$$

etc. By induction, it can be shown that

$$
\mathbf{B}_{r}=\left[\begin{array}{cc}
F_{n+r+2} & F_{n+2} \\
a^{2} F_{n+r+1} & a^{2} F_{n+1}
\end{array}\right]
$$

From the determinant sum property, we obtain

$$
\left|\mathbf{B}_{r}\right|=a z\left|\mathbf{B}_{r-1}\right|+a^{2}\left|\mathbf{B}_{r-2}\right| .
$$

The recurrence relation (27) implies that the sequence $\left\{\left|\mathbf{B}_{r}\right|\right\}$ is actually a generalized Fibonacci polynomial sequence. It is easily seen that $\left|\mathbf{B}_{0}\right|=0$ and from Corollary 16, we get

$$
\left|\mathbf{B}_{1}\right|=(-1)^{n+2} a^{2 n+4}
$$


By the relation (27) and the Definition 1, we obtain

$$
\left|\mathbf{B}_{r}\right|=(-1)^{n+2} a^{2 n+4} F_{r} .
$$

On the other hand, the determinant of matrix (26) is

$$
\left|\mathbf{B}_{r}\right|=a^{2}\left(F_{n+r+2} F_{n+1}-F_{n+r+1} F_{n+2}\right) .
$$

By writing $m=n+r$ in (29) and using (28), we have

$$
F_{m+2} F_{n+1}-F_{m+1} F_{n+2}=(-1)^{n+2} a^{2 n+2} F_{m-n}
$$

and by replacing $m$ and $n$ with $m-1$ and $n-1$ in (30), respectively,

$$
F_{m+1} F_{n}-F_{m} F_{n+1}=(-1)^{n+1} a^{2 n} F_{m-n} .
$$

The identity (25) follows from last identity and the proof is complete.

Theorem 19 (Generalized Catalan's Identity). For $m \geqslant n \geqslant 1$,

$$
F_{n} F_{m}-F_{n-r} F_{m+r}=(-1)^{n-r} a^{2 n-2 r} F_{m-n+r} F_{r} .
$$

Proof. Let $\mathbf{C}_{0}$ be the matrix

$$
\mathbf{C}_{0}=\left[\begin{array}{cc}
F_{n} & F_{n-r} \\
a^{2} F_{n+1} & a^{2} F_{n+1}
\end{array}\right]
$$

and

$$
\mathbf{C}_{1}=\left[\begin{array}{ll}
F_{n+1} & F_{n-r+1} \\
a^{2} F_{n} & a^{2} F_{n-r}
\end{array}\right] .
$$

be the matrix obtained by replacing $n$ with $n-r-1$ in the matrix $\mathbf{B}_{r}$. We now construct the matrix $\mathbf{C}_{s}$ by adding the first row of the matrix $\mathbf{C}_{s-2}$ multiplied with $a^{2}$ to the first row of the matrix $\mathbf{C}_{s-1}$ multiplied with $a z$, for $s \geqslant 2$. By induction, it can be shown that

$$
\mathbf{C}_{s}=\left[\begin{array}{ll}
F_{n+s} & F_{n-r+s} \\
a^{2} F_{n} & a^{2} F_{n-r}
\end{array}\right] \text {. }
$$

From the determinant sum property, we obtain

$$
\left|\mathbf{C}_{s}\right|=a z\left|\mathbf{C}_{s-1}\right|+a^{2}\left|\mathbf{C}_{s-2}\right| .
$$

Th recurrence relation (33) implies that the sequence $\left\{\left|\mathbf{C}_{s}\right|\right\}$ is also a generalized Fibonacci polynomial sequence. It is obvious that $\left|\mathbf{C}_{0}\right|=0$ and by $(28)$, we get $\left|\mathbf{C}_{1}\right|=$ $(-1)^{n-r+1} a^{2 n-2 r+2} F_{r}$. By induction on $s$ in the relation (33) and Definition 1 we get

$$
\left|\mathbf{C}_{s}\right|=(-1)^{n-r+1} a^{2 n-2 r+2} F_{r} F_{s} .
$$

On the other hand, the determinant of matrix (32) is

$$
\left|\mathbf{C}_{s}\right|=a^{2}\left(F_{n-r} F_{n+s}-F_{n-r+s} F_{n}\right) .
$$

By writing $s=m-n+r$ in (35) and using (34), we have

$$
F_{n-r} F_{m+r}-F_{m} F_{n}=(-1)^{n-r+1} a^{2 n-2 r} F_{m-n+r} F_{r} .
$$

Thus, the identity (31) is obtained from the identity (36) and the proof is complete. 
Remark 20. For $m=n$ in the identity (31), we have Catalan's identity

$$
F_{n}^{2}-F_{n-r} F_{n+r}=(-1)^{n-r} a^{2 n-2 r} F_{r}^{2}
$$

for $F_{n}(a, z)$. The Cassini's identity $(24)$ is a special case of Catalan's identity with $r=1$.

Definition 21. Let $\mathbf{D}$ denotes the matrix of size $2 \times 2$ which is associated with $P_{n}(a, z)$ as

$$
\mathbf{D}=\left[\begin{array}{cc}
a z+\left(a-a^{3}\right) z^{-1} & 1 \\
a^{2} & \left(a-a^{3}\right) z^{-1}
\end{array}\right]
$$

Note that, $\mathbf{D}=\mathbf{A}+\left(a-a^{3}\right) z^{-1} \mathbf{I}$.

Theorem 22. For $n \geqslant 2$,

$$
\boldsymbol{D A}^{n-1}=\left[\begin{array}{cc}
P_{n+1} & P_{n} \\
a^{2} P_{n} & a^{2} P_{n-1}
\end{array}\right]
$$

where $P_{n}=P_{n}(a, z)$ denotes the HOMFLY polynomial of $(2, n)$-torus link.

Proof. By matrix product and considering (5), we have

$$
\mathbf{D A}^{n-1}=\left[\begin{array}{cc}
F_{n+1}+\left(a-a^{3}\right) z^{-1} F_{n} & F_{n}+\left(a-a^{3}\right) z^{-1} F_{n-1} \\
a^{2}\left(F_{n}+\left(a-a^{3}\right) z^{-1} F_{n-1}\right) & a^{2}\left(F_{n-1}+\left(a-a^{3}\right) z^{-1} F_{n-2}\right)
\end{array}\right] .
$$

From Theorem 12, we obtain the matrix (37).

Corollary 23 (Cassini's Identity). For $n \geqslant 2$,

$$
P_{n+1} P_{n-1}-P_{n}^{2}=(-1)^{n-1}\left(\left(1-a^{2}\right)^{2} z^{-2}-a^{2}\right) a^{2 n-2} .
$$

Proof. By using $\left|\mathbf{D A}^{n-1}\right|=|\mathbf{D}|\left|\mathbf{A}^{n-1}\right|$, we get result.

Corollary 24. For $n, m \geqslant 0$,

$$
P_{n+m+1}+\left(a-a^{3}\right) z^{-1} P_{n+m}=P_{n+1} P_{m+1}+a^{2} P_{n} P_{m}
$$

and in particular, for $n=m$,

$$
P_{2 n+1}+\left(a-a^{3}\right) z^{-1} P_{2 n}=P_{n+1}^{2}+a^{2} P_{n}^{2} .
$$

Proof. Since $\mathbf{D} A^{n}=\mathbf{A}^{n} \mathbf{D}$, this results are obtained from $\mathbf{D}\left(\mathbf{D} \mathbf{A}^{n+m}\right)=\left(\mathbf{D A}^{n}\right)\left(\mathbf{D} \mathbf{A}^{m}\right)$.

Theorem 25 (d'Ocagne-like Identity). For $m \geqslant n \geqslant 2$,

$$
P_{n+1} P_{m}-P_{n} P_{m+1}=(-1)^{n+1} a^{2 n}\left(\left(1-a^{2}\right)^{2} z^{-2}-a^{2}\right) F_{m-n} .
$$


Proof. Let $\mathbf{E}_{0}$ be the matrix

$$
\mathbf{E}_{0}=\left[\begin{array}{cc}
P_{n+2} & P_{n+2} \\
a^{2} P_{n+1} & a^{2} P_{n+1}
\end{array}\right] .
$$

We construct the matrix $\mathbf{E}_{1}$ by adding the first column of the matrix $\mathbf{A}^{n-1}$ multiplied with $a^{2}$ to the first column of the matrix $\mathbf{E}_{0}$ multiplied with $a z$. Thus, we have

$$
\mathbf{E}_{1}=\left[\begin{array}{cc}
P_{n+3} & P_{n+2} \\
a^{2} P_{n+2} & a^{2} P_{n+1}
\end{array}\right]
$$

We now construct the matrix $\mathbf{E}_{r}$ by adding the first column of the matrix $\mathbf{E}_{r-2}$ multiplied with $a^{2}$ to the first column of the matrix $\mathbf{E}_{r-1}$ multiplied with $a z$, for $r \geqslant 2$. By induction, it can be shown that

$$
\mathbf{E}_{r}=\left[\begin{array}{cc}
P_{n+r+2} & P_{n+2} \\
a^{2} P_{n+r+1} & a^{2} P_{n+1}
\end{array}\right]
$$

From the determinant sum property, we obtain

$$
\left|\mathbf{E}_{r}\right|=a z\left|\mathbf{E}_{r-1}\right|+a^{2}\left|\mathbf{E}_{r-2}\right| .
$$

The recurrence relation (41) implies that the sequence $\left\{\left|\mathbf{E}_{r}\right|\right\}$ is a generalized Fibonacci polynomial sequence. It is easily seen that $\left|\mathbf{E}_{0}\right|=0$ and from Corollary 23, we get

$$
\left|\mathbf{E}_{1}\right|=(-1)^{n+1} a^{2 n+4}\left(\left(1-a^{2}\right)^{2} z^{-2}-a^{2}\right) .
$$

By the relation (41) and Definition 1, we obtain

$$
\left|\mathbf{E}_{r}\right|=(-1)^{n+1} a^{2 n+2}\left(\left(1-a^{2}\right)^{2} z^{-2}-a^{2}\right) F_{r} .
$$

On the other hand, the determinant of matrix (40) is

$$
\left|\mathbf{E}_{r}\right|=a^{2}\left(P_{n+r+2} P_{n+1}-P_{n+r+1} P_{n+2}\right) .
$$

By writing $m=n+r$ in (43) and using (42), we have

$$
P_{m+2} P_{n+1}-P_{m+1} P_{n+2}=(-1)^{n+1} a^{2 n+2}\left(\left(1-a^{2}\right)^{2} z^{-2}-a^{2}\right) F_{m-n}
$$

and by replacing $m$ and $n$ with $m-1$ and $n-1$ in the identity (44), respectively,

$$
P_{m+1} P_{n}-P_{m} P_{n+1}=(-1)^{n} a^{2 n}\left(\left(1-a^{2}\right)^{2} z^{-2}-a^{2}\right) F_{m-n} .
$$

Thus, the identity (39) is obtained from last identity and the proof is complete.

Theorem 26 (Generalized Catalan-like Identity). For $m \geqslant n \geqslant 1$,

$$
P_{n} P_{m}-P_{n-r} P_{m+r}=(-1)^{n-r+1} a^{2 n-2 r}\left(\left(1-a^{2}\right)^{2} z^{-2}-a^{2}\right) F_{m-n+r} F_{r} .
$$


Proof. Let $\mathbf{F}_{0}$ be the matrix

$$
\mathbf{F}_{0}=\left[\begin{array}{cc}
P_{n} & P_{n-r} \\
a^{2} P_{n+1} & a^{2} P_{n+1}
\end{array}\right]
$$

and

$$
\mathbf{F}_{1}=\left[\begin{array}{ll}
P_{n+1} & P_{n-r+1} \\
a^{2} P_{n} & a^{2} P_{n-r}
\end{array}\right]
$$

be the matrix obtained by replacing $n$ with $n-r-2$ in the matrix $\mathbf{E}_{r}$. We now construct the matrix $\mathbf{F}_{s}$ by adding the first row of the matrix $\mathbf{F}_{s-2}$ multiplied with $a^{2}$ to the first row of the matrix $\mathbf{F}_{s-1}$ multiplied with $a z$, for $s \geqslant 2$. By induction, it can be shown that

$$
\mathbf{F}_{s}=\left[\begin{array}{ll}
P_{n+s} & P_{n-r+s} \\
a^{2} P_{n} & a^{2} P_{n-r}
\end{array}\right]
$$

From the determinant sum property, we obtain

$$
\left|\mathbf{F}_{s}\right|=a z\left|\mathbf{F}_{s-1}\right|+a^{2}\left|\mathbf{F}_{s-2}\right| .
$$

The recurrence relation (47) implies that the sequence $\left\{\left|\mathbf{F}_{s}\right|\right\}$ is also a generalized Fibonacci polynomial sequence. It is obvious that $\left|\mathbf{F}_{0}\right|=0$ and by (42), we get

$$
\left|\mathbf{F}_{1}\right|=(-1)^{n-r+1} a^{2 n-2 r+2}\left(\left(1-a^{2}\right)^{2} z^{-2}-a^{2}\right) F_{r} .
$$

By induction on $s$ in the relation (47) and Definition 1, we have

$$
\left|\mathbf{F}_{s}\right|=(-1)^{n-r+1} a^{2 n-2 r+2}\left(\left(1-a^{2}\right)^{2} z^{-2}-a^{2}\right) F_{r} F_{s} .
$$

The determinant of the matrix (46) is

$$
\left|\mathbf{F}_{s}\right|=a^{2}\left(P_{n-r} P_{n+s}-P_{n-r+s} P_{n}\right) .
$$

By writing $s=m-n+r$ in (49) and using (48), we obtain

$$
P_{n-r} P_{m+r}-P_{m} P_{n}=(-1)^{n-r} a^{2 n-2 r}\left(\left(1-a^{2}\right)^{2} z^{-2}-a^{2}\right) F_{m-n+r} F_{r} .
$$

Thus, the identity (45) is obtained from last identity and the proof is complete.

Remark 27. For $m=n$ in the identity (45), we have

$$
P_{n}^{2}-P_{n-r} P_{n+r}=(-1)^{n-r+1} a^{2 n-2 r}\left(\left(1-a^{2}\right)^{2} z^{-2}-a^{2}\right) F_{r}^{2},
$$

which is a Catalan-like identity. The Cassini's identity (38) is a special case of Catalan-like identity with $r=1$. 


\section{References}

[1] J. W. Alexander. Topological invariants of knots and links. Trans. Amer. Math. Soc., 30:275-308, 1928.

[2] İ. Altıntaş. An oriented state model for the Jones polynomial and its applications alternating links. Applied Math. and Comp., 194(1):168-178, 2007.

[3] T. Amdeberhan, V.H. Moll, X. Chen, and B.E. Sagan. Generalized Fibonacci polynomials and fibonomial coefficients. Annals of Combin., 18(4):541-562, 2014.

[4] A. T. Benjamin, N. T. Cameron, and J. J. Quinn. Fibonacci determinants - a combinatorial approach. Fibonacci Quart., 45(1):39-55, 2007.

[5] R. D. Brandt, W. B. R. Lickorish, and K. C. Millett. A polynomial invariant for unoriented knots and links. Invent. Math., 84:563-573, 1986.

[6] M. Catalani. Generalized bivariate Fibonacci polynomials. Preprint, 2004. arXiv:math/0211366v2

[7] J. H. Conway. An enumeration of knots and links, and some of their algebraic properties. In: Computational Problems in Abstract Algebra (Proc. Conf., Oxford, 1967), pages 329-358, Oxford, 1970. Pergamon.

[8] R. H. Crowell and R. H. Fox. Introduction to Knot Theory, volume 57 of Graduate Texts in Mathematics. Springer, 1963.

[9] J. Feng. Fibonacci identities via the determinant of tridiagonal matrix. Applied Math. and Comp., 217:5978-5981, 2011.

[10] R. H. Fox. Free differential calculus I: Derivation in the free group ring. Annals of Math., 57(3):547-560, 1953.

[11] P. Freyd, D. Yetter, J. Hoste, W. B. R. Lickorish, K. Millett, and A. Ocneau. A new polynomial invariant of knots and links. Bull. Amer. Math. Soc., 12:239-246, 1985.

[12] H.W. Gould. Combinatorial Identities. Morgantown, W Va, 1972.

[13] R. L. Graham, D. E. Knuth, and O. Patashnik. Concrete Mathematics: A Foundation for Computer Science. Addison-Wesley, 1994.

[14] V. F. R. Jones. A polynomial invariant for knots via von Neumann algebras. Bull. Amer. Math. Soc., 12:103-111, 1985.

[15] L. H. Kauffman. On Knots, volume 115 of Annals of Mathematics Study. Princeton University Press, 1987.

[16] L. H. Kauffman. State models and the Jones polynomial. Topology, 26(3):395-407, 1987.

[17] L. H. Kauffman. New invariants in the theory of knots. Amer. Math. Monthly, 95(3):195-242, 1988.

[18] L. H. Kauffman. An invariant of regular isotopy. Trans. Amer. Math. Soc., 318:417471, 1990. 
[19] P.-V. Koseleff and D. Pecker. On Fibonacci knots. Fibonacci Quart., 48(2):137-143, 2010.

[20] T. Koshy. Fibonacci and Lucas Numbers with Applications. Pure and Applied Mathematics: A Wiley Series of Texts, Monographs and Tracts. Wiley, 2011.

[21] K. Murasugi. Knot Theory and Its Applications. Birkhäuser, Basel, 1996.

[22] A. Nalli and P. Haukkanen. On generalized Fibonacci and Lucas polynomials. Chaos, Solitons and Fractals, 42(5):3179-3186, 2009.

[23] Y. K. Panwar and M. Singh. Generalized bivariate Fibonacci-like polynomials. Inter. Journal of Pure Math., 1:8-13, 2014.

[24] M. Z. Spivey. Fibonacci identities via the determinant sum property. The College Math. Journal, 37(4):286-289, 2006. 